\title{
Quantum entanglement and the self-trapping transition in polaronic systems
}

\author{
Yang Zhao, ${ }^{1}$ Paolo Zanardi, ${ }^{2}$ and Guanhua Chen ${ }^{1}$ \\ ${ }^{1}$ Department of Chemistry, University of Hong Kong, Pokfulam Road, Hong Kong, People's Republic of China \\ ${ }^{2}$ Institute for Scientific Interchange, Villa Gualino, Viale Settimio Severo 65, I-10133 Torino, Italy
}

(Received 14 July 2004; published 17 November 2004)

\begin{abstract}
We revisit from a quantum-information perspective a classic problem of polaron theory in one dimension. In the context of the Holstein model we show that a simple analysis of quantum entanglement between excitonic and phononic degrees of freedom allows one to effectively characterize both the small and large polaron regimes as well as the crossover in between. The small (large) polaron regime corresponds to a high (low) degree of bipartite quantum entanglement between the exciton and the phonon cloud that clothes the exciton. Moreover, the self-trapping transition is clearly displayed by a sharp drop of exciton-phonon entanglement.
\end{abstract}

DOI: 10.1103/PhysRevB.70.195113

PACS number(s): 71.38.Ht, 03.67.Mn

\section{INTRODUCTION}

Almost three quarters of a century have passed since the concept of polaronic self-trapping was first conceived by Landau, ${ }^{1,2}$ and yet many questions remain unanswered regarding some of the most simplistic polaron Hamiltonians. In this paper we will approach this classic problem from the contemporary point of view of quantum entanglement. ${ }^{3}$ The latter concept plays a central role in the burgeoning field of quantum information in that quantum entanglement represents the key physical resource at the basis of quantum information protocols. ${ }^{4}$ More recently, a growing amount of attention has been devoted to analyses of quantum entanglement in many-body systems undergoing quantum phase transitions. ${ }^{5}$

An emerging picture is that various entanglement measures, such as the two-qubit concurrence and the entanglement entropy of a subsystem, provide different, often complementary physical insights onto the nature of manybody wave functions. Tools drawn from quantum information theory provide a deeper, previously unavailable understanding of quantum correlations and their qualitative changes across boundaries of different phase-diagram regimes in these many-body systems. Moreover, inspired by ideas from quantum-information theory, efficient computational schemes for studying quantum many-body systems have been devised. ${ }^{6}$

The study to be carried out in this paper aims to exemplify the ongoing efforts bridging the field of quantum information theory and established areas of condensed matter physics. We will show that an analysis of quantum entanglement between electronic (or excitonic) and phononic degrees of freedom in a polaronic model allows one to characterize the polaron phase diagram in a strikingly simple fashion. In particular, the self-trapping transition, i.e., a crossover between the so-called small and large polaron regimes, is readily captured by the behavior of the linear entropy of the excitonic reduced density matrix. We will show that the small (large) polaron regime corresponds to a high (low) degree of quantum entanglement between the exciton and its phononic environment. Moreover, the self-trapping transition is clearly displayed by a sharp drop of exciton-phonon entanglement.
It is worthwhile to stress that, at variance with most of the other studies of this type, the particular form of quantum entanglement we are going to analyze is between systems of distinct physical nature: the exciton, a finite-dimensional system, and the phonons, a bosonic bath. To emphasize this fact we will refer to the bipartite quantum entanglement between the exciton and its phonon environment in the polaron problem as heteroentanglement. This situation bears resemblance to those in decoherence studies where a system under examination, e.g., a qubit, is coupled with its environmental degrees of freedom, which spoils the purity of the system state. From this point of view, the polaronic entanglement to be analyzed in this paper can be viewed as a measure of the decoherence of the excitonic (phononic) state induced by the coupling with the lattice phonons (excitons).

This paper is structured as follows. In Sec. II, the Holstein model is introduced, and the corresponding phase diagram of polaronic self-trapping is presented. In Sec. III, we evaluate heteroentanglement between the exciton and the phonon bath, and examine its relation to polaronic self-trapping. Discussions are given in Sec. IV, in which we show that excitonic superradiance, viewed as a form of intraexciton entanglement between spatially distinct excitonic modes, complement the exciton-phonon entanglement.

\section{SELF-TRAPPING AND PHASE DIAGRAM OF THE HOLSTEIN MODEL}

We first introduce a Frenkel-exciton model Hamiltonian, also known as the Holstein molecular crystal model, which describes a lattice of two-level molecules interacting with a bath consisting of nuclear (intramolecular, intermolecular, and solvent) degrees of freedom

$$
\hat{H}=\sum_{n} \Omega_{n}(\mathbf{q}) B_{n}^{\dagger} B_{n}+\sum_{m, n}^{m \neq n} J_{m n}(\mathbf{q}) B_{m}^{\dagger} B_{n}+\hat{H}^{\mathrm{ph}} .
$$

Here $B_{n}\left(B_{n}^{\dagger}\right)$ are exciton annihilation (creation) operators for the $n$th molecule, $\hat{H}^{\text {ph }}$ is the bath (phonon) Hamiltonian, and q represents the complete set of nuclear coordinates. The excitonic operators satisfy the hard-core bosons relations $\left(B_{n}^{\dagger}\right)^{2}=B_{n}^{2}=0,\left[B_{n} B_{m}^{\dagger}\right]=\delta_{n m}$. It follows that each site label $n$ is 
associated with a two-level system, i.e., a qubit. Notice that in terms of the Pauli matrices one has $B^{\dagger}=\sigma^{+}$, and $B=\sigma^{-}$. Despite that this latter notation is the standard one in quantum information, in order to keep in line with the vast polaronic literature, we will stick to the $B$ operators. Interested readers should not have any problem in translating the formulas to the Pauli matrices notation.

Exciton-phonon interactions originate from dependence of molecular frequencies $\Omega_{n}$ and the intermolecular couplings $J_{m n}$ on nuclear coordinates q. We adopt the Hamiltonian Eq. (1) with the Einstein phonon Hamiltonian $\hat{H}^{\text {ph }}$ $=\sum_{n} \hbar \omega_{0} b_{n}^{\dagger} b_{n}$, where $b_{n}^{\dagger}$ creates a phonon of frequency $\omega_{0}$ on site $n$, and we have one Einstein oscillator per molecule. Exciton-phonon interactions enter through the nuclear coordinate influence on both molecular frequencies (diagonal coupling) and intermolecular interactions (off-diagonal coupling). Expanding $\Omega_{n}(\mathbf{q})$ to first order in phonon coordinate $\mathbf{q}$, the first term of Eq. (1) reads $\sum_{n} \Omega_{n}(\mathbf{q}) B_{n}^{\dagger} B_{n}=\sum_{n} \Omega_{n}(\mathbf{q}$ $=0) B_{n}^{\dagger} B_{n}+\hat{H}^{\text {diag }}$ with the diagonal exciton-phonon coupling term

$$
\hat{H}^{\mathrm{diag}}=g \hbar \omega_{0} \sum_{n} B_{n}^{\dagger} B_{n}\left(b_{n}^{\dagger}+b_{n}\right),
$$

and $g$ is a dimensionless diagonal coupling constant. Expanding $J_{m n}(\mathbf{q})$ to first order in phonon coordinates, we write the second term of Eq. (1) as, for example, $\Sigma_{m \neq n} J_{m n}(\mathbf{q}) B_{m}^{\dagger} B_{n}=\Sigma_{m \neq n} J_{m n}(\mathbf{q}=0) B_{m}^{\dagger} B_{n}+\hat{H}^{\text {o.d. }}$ with the transfer integral $J_{m n}(\mathbf{q}=0)=-J \delta_{n, m \pm 1}$ and the off-diagonal coupling term ${ }^{7,8}$

$$
\begin{aligned}
\hat{H}^{\text {o.d. }}= & \frac{1}{2} \phi \hbar \omega_{0} \sum_{n l}\left[B_{n}^{\dagger} B_{n+1}\left(b_{l}^{\dagger}+b_{l}\right)\left(\delta_{n+1, l}-\delta_{n l}\right)\right. \\
& \left.+B_{n}^{\dagger} B_{n-1}\left(b_{l}^{\dagger}+b_{l}\right)\left(\delta_{n l}-\delta_{n-1, l}\right)\right] .
\end{aligned}
$$

The second term of (3) is the Hermitian conjugate of the first, and we have assumed nearest-neighbor coupling of the antisymmetric type with $\phi$ a dimensionless parameter controlling the off-diagonal coupling strength. Off-diagonal coupling may adopt various forms ${ }^{8,9}$ other than the antisymmetric type (3), and can play important roles in electronic properties of solid. In the theory of high-temperature superconductivity, for example, it has been recently proposed ${ }^{9}$ that off diagonal coupling modulates the hopping integral of the Zhang-Rice singlet and the superexchange interaction, and is especially relevant in the low-doping regime. Equations (2) and (3), together with $\hat{H}^{\text {ph }}$ and the zeroth-order intermolecular coupling term, result in the generalized Holstein Hamiltonian $\hat{H}^{\mathrm{GH}}$ (The original Holstein Hamiltonian contains diagonal coupling only. $)^{10}$

$$
\begin{aligned}
\hat{H}^{\mathrm{GH}}= & \sum_{n} \Omega_{n}(\mathbf{q}=0) B_{n}^{\dagger} B_{n}+\hat{H}^{\mathrm{diag}} \\
& +\sum_{m n}^{m \neq n} J_{m n}(\mathbf{q}=0) B_{m}^{\dagger} B_{n}+\hat{H}^{\text {o.d. }}+\hat{H}^{\mathrm{ph}} .
\end{aligned}
$$

There are two competing energy scales in the Holstein Hamiltonian in the absence of off-diagonal exciton-phonon coupling, namely, the lattice relaxation energy $g^{2} \omega_{0}$ and the bare exciton bandwidth $4 J$. Their ratio will be denoted the coupling strength $\kappa=g^{2} \omega_{0} / 4 J$ which determines the size of the polaron as well as exciton-phonon correlations. In typical molecular crystals, $g^{2} \leqslant 1$, in ionic crystals, $g^{2}$ is large compared to unity, and in semiconductors, $g^{2}$ is between the former two. In anthracence, for example, $\kappa$ is about 0.4 , and in pyrene, about $0.8-1.6 .{ }^{11}$ For strong exciton-phonon coupling $(\kappa \gg 1)$, solutions of the Holstein Hamiltonian are known as small polarons because the exciton-induced lattice distortion is confined to essentially a single exciton site. ${ }^{12}$ For weak exciton-phonon coupling $(\kappa \ll 1)$, the spatial extent of the lattice distortion is significantly increased and the resulting phonon-dressed exciton is called a large polaron. The crossover from a large polaron to a small polaron with increasing exciton-phonon coupling (essentially often called the self-trapping transition) is rather abrupt for large intermolecular coupling $J$. In the limit of slow lattice motions, adiabatic polaron theories admit approximate solutions in the form of solitons.

The one-dimensional Holstein Hamiltonian with diagonal and off-diagonal coupling to Einstein phonons has been previously ${ }^{13-16}$ modeled by a variational wave function pioneered by Toyozawa (labeled as the Toyozawa Ansatz in Ref. 15)

$$
|K\rangle=N^{-1} \sum_{n} e^{i K n}\left|\Lambda_{n}^{K}\right\rangle \sum_{m} \psi_{m-n}^{K} B_{m}^{\dagger}|0\rangle_{e} .
$$

Here $|K\rangle$ is the lowest energy polaron state with momentum $K,|0\rangle_{\mathrm{e}}$ is the exciton vacuum state, and $\left|\Lambda_{n}^{K}\right\rangle$ are phonon wave functions centered at site $n$ containing a coherent state on each site $n_{1}$ with a displacement $\lambda_{n_{1}-n}^{K}$ :

$$
\left|\Lambda_{n}^{K}\right\rangle=\exp \left[-\sum_{n_{1}}\left(\lambda_{n_{1}-n}^{K} b_{n_{1}}^{\dagger}-\lambda_{n_{1}-n}^{K^{*}} b_{n_{1}}\right)\right]|0\rangle_{\mathrm{ph}} .
$$

$|0\rangle_{\text {ph }}$ is the phonon vacuum state, and $\left|\Lambda_{n}^{K}\right\rangle$ is different from $\left|\Lambda_{n^{\prime}}^{K}\right\rangle$ only by a shift of $n-n^{\prime}$ lattice constants. The parameters $\lambda_{l}^{K}$ and $\psi_{l}^{K}$ are obtained variationally. The phonon wave functions $\left|\Lambda_{n}^{K}\right\rangle$ represent a lattice distortion forming a potential well centered at $n$ and trapping the exciton with an amplitude distribution of $\psi_{l}^{K}$. The Toyozawa Ansatz state (5) is not normalized: $\langle K \mid K\rangle=\Sigma_{n m} e^{-i K n} \psi_{m}^{K} \psi_{m-n}^{K^{*}} S_{n}^{K}$ where $S_{n}^{K}$ is the Debye-Waller factor: $S_{n}^{K} \equiv\left\langle\Lambda_{m}^{K} \mid \Lambda_{m-n}^{K}\right\rangle=\exp \left[N^{-1} \Sigma_{q}\left|\lambda_{q}^{K}\right|^{2}\left(e^{i q n}\right.\right.$ $-1)$ ], and $\lambda_{q}^{K}$ are the Fourier transform of $\lambda_{n}^{K}$. The variational methods are shown to be rather efficient while remaining quantitatively accurate compared with calculations involving far more expensive computational resources. ${ }^{17,18}$

We introduce the phonon-traced exciton density matrix $\rho_{e}^{K}$ for the state $|K\rangle$ :

$$
\rho_{e}^{K}:=\langle K \mid K\rangle^{-1} \operatorname{Tr}_{\mathrm{ph}}(|K\rangle\langle K|),
$$

where $\operatorname{Tr}_{\mathrm{ph}}$ stands for tracing over the phonon degrees of freedom. To calculate $\rho_{e}^{K}$ we assume that exciton-phonon coupling leads to the formation of bands of collective exciton-phonon states, and the many-body polaron wave 


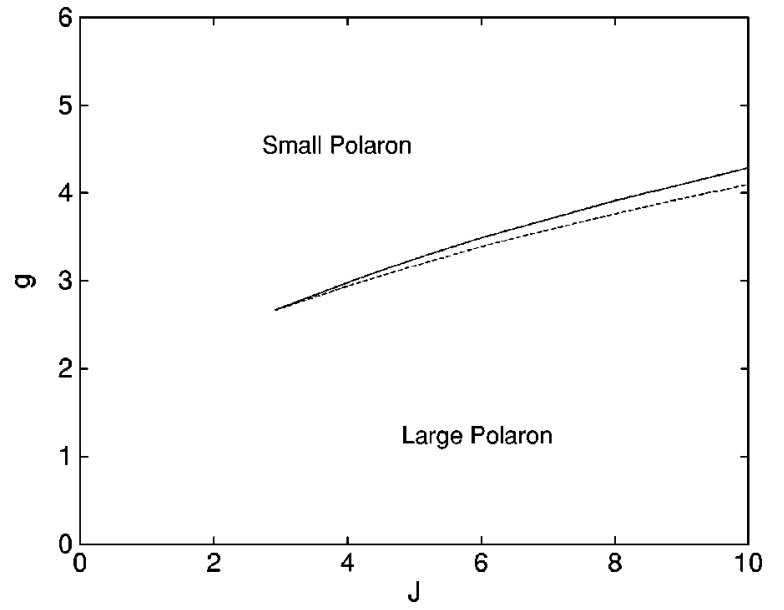

FIG. 1. The $J$ - $g$ phase diagram for the Toyozawa Ansatz. A thin sword-shaped regime identifies with the polaronic self-trapping line, across which the effective mass of the polaron decreases drastically as $g$ is decreased. The top (solid) line of the sword-shaped regime indicates the onset of bifurcation at $K=0$ when the polaronic structure goes through a sudden change as one travels vertically downward in the phase diagram, and the bottom (dashed) line indicates where the state of the highest $K$ (in the vicinity of $K=0$ ) for which a discontinuous change in the polaronic structure is observed acquires two solutions as one travels vertically upward.

function for the ground state band is given by the Toyozawa Ansatz ${ }^{13-15}$

$$
\left(\rho_{e}^{K}\right)_{m m^{\prime}}=N^{-1}\langle K \mid K\rangle^{-1} \sum_{n n^{\prime}} e^{i K\left(n^{\prime}-n\right)} \psi_{m-n}^{K} \psi_{m^{\prime}-n^{\prime}}^{K^{*}} S_{n^{\prime}-n}^{K} .
$$

In this paper we confine our attention to the case of diagonal exciton-phonon coupling only. Localization of $\left(\rho_{e}^{K=0}\right)_{m m^{\prime}}$ is determined by the combined effect of electronic confinement $\psi_{n}^{K=0}$ and the overlap of the adjacent nuclear wave functions $S_{n}^{K=0}$. An efficient iterative procedure is employed to identify optimized $\lambda_{l}^{K}$ and $\psi_{l}^{K} .{ }^{14}$ Computation typically starts from the $J=0$ axis where exact solutions exist. A convergent solution at one point in the phase diagram is used to initiate a variation at a neighboring point. Reversibility of the calculation and uniqueness of the solution are checked via altering initializations of the iterative procedure as the parameter space is mapped. A phase diagram for the Toyozawa Ansatz spanned by $J$ and $g$ is presented in Fig. 1 which displays a thin sword-shaped region identified with the polaronic self-trapping. The top (solid) line of the swordshaped regime indicates the onset of bifurcation at $K=0$ when the polaronic structure goes through a sudden change as one travels vertically downward in the phase diagram, and the bottom (dashed) line indicates where the state of the highest $K$ (in the vicinity of $K=0$ ), for which a discontinuous change in the polaron structure is observed, acquires two solutions as one travels vertically upward. Outside the sword-shaped area, solutions to self-consistent variational equations are unique and independent of how the iterative procedure is initialized. The polaronic structure for $(J, g)$ points above (below) the sword-shaped area is traditionally identified as small (large) polarons.
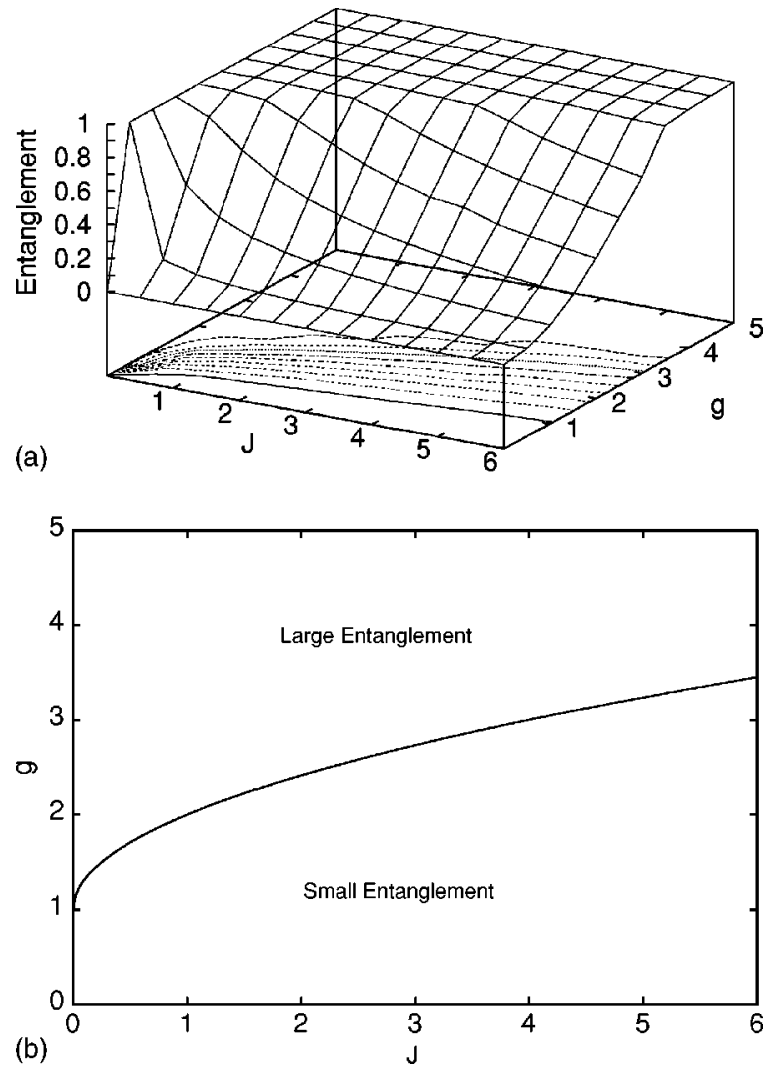

FIG. 2. Heteroentanglement between the exciton and the phonons as measured by the linear entropy $1-\operatorname{Tr}_{e}\left[\left(\rho_{e}^{K=0}\right)^{2}\right]$ is displayed in the upper panel for the entire $J-g$ phase diagram. The entanglement is calculated for the lowest energy state with zero crystal momentum $K=0$. The solid line in the lower panel is the edge of the cliff which separates the large and small entanglement regions.

\section{HETEROENTANGLEMENT AND POLARONIC SELF-TRAPPING}

For a finite bare exciton band width $J>0$, the lowest energy state for diagonal coupling only has zero crystal momentum $K=0$. In this lowest-energy $K=0$ state, heteroentanglement between the two species in the Holstein Hamiltonian, the exciton and the phonons, as measured by the linear entropy, has the form

$$
E:=1-\operatorname{Tr}_{e}\left[\left(\rho_{e}^{K=0}\right)^{2}\right] .
$$

The function (8) is a linearized version of the von Neumann entropy $S(\rho)=-\operatorname{Tr} \rho \ln \rho$; it shares with this latter quantity the properties: (i) $E=0 \Leftrightarrow \rho=\rho^{2}$, i.e., $\rho$ is a pure state; (ii) $E_{\max }$ $=1-1 / D=E(1 / D)$, i.e., the linear entropy is at its maximum for the totally mixed state $1 / D(D=$ is the dimension of the space). Moreover, for qubits, the linear and von Neumann entropy are monotonic functions of each other. The linear entropy (8) has a close relation with the so-called 2-Renyi entropy. ${ }^{19}$ These considerations show that (8) represent a good choice as a simple entanglement measure. Calculated heteroentanglement is shown in Fig. 2 for the entire $J$ vs $g$ phase diagram. It is important to note that the qualitative behavior of the ground state entanglement as a function of 
control parameters discussed above does not depend on the specific choice of the linear entropy as the entanglement measure; analogous calculations performed with the von Neumann entropy give rise to the very same qualitative picture. $^{20}$

On the $J=0$ vertical axis, the exact wave function of the Holstein polaron can be written as $\Sigma_{n} B_{n}^{\dagger}|0\rangle_{e}\left|\Lambda_{n}^{J=0}\right\rangle$, where $\left\langle\Lambda_{m}^{J=0} \mid \Lambda_{n}^{J=0}\right\rangle=\delta_{m n}$. Therefore, the heteroentanglement between the two species reaches its maximum on the $J=0$ vertical axis: $E_{\max }=1-N^{-1}$. On the horizontal axis $g=0$, on the contrary, the $K=0$ state is separable with respect to the two species.

As shown in the upper panel of Fig. 2, exciton-phonon heteroentanglement forms a cliff. The edge of the cliff is found overlapping with the thin sword-shaped area in Fig. 1 (i.e., the self-trapping line), and can be fitted empirically by $J_{c}=\left(g_{c}-1\right)^{2} \hbar \omega_{0}$ : on the cliff plateau, $E$ approaches $1-N^{-1}$; on the other side of the cliff edge, $E$ decreases rapidly to zero with increasing $J$ or decreasing $g$. In the lower panel, the solid line is the projection of the cliff edge onto the $J$ - $g$ plane which separates the large and small entanglement phases. The usual distinction between the small and large polarons can thus be rephrased as follows: the small polaron is a maximally entangled exciton-phonon entity, while the large polaron has much-reduced exciton-phonon heteroentanglement. The heteroentanglement is therefore a good measure of large and small polarons and the transition in between.

\section{DISCUSSIONS}

In this paper we have revisited the classic polaron problem utilizing the concept of quantum entanglement. The exciton-phonon coupling, described by the Holstein Hamiltonian, induces strong quantum coherences between the two heterogeneous physical degrees of freedom. These coherences can be conveniently quantified by the linear entropy of the excitonic (or phononic) reduced density matrix. The latter has been explicitly computed by employing the Toyozawa Ansatz. Difficulties associated to the infinite dimensionality of the phonon degrees of freedom can be circumvented by using the Toyozawa variational Ansatz to approximate the ground-state polaronic wave function, for which an exact form remains elusive. This choice of Ansatz states also allows us to carry out a large portion of the calculations in an analytical, conceptually transparent fashion. The study of the entanglement behavior, as a function of the controlling parameters $J$ and $g$ (exciton hopping amplitude and excitonphonon coupling, respectively), allows a very simple characterization of the zero-temperature phase diagram of the model. The self-trapping transition from large to small po- laron regimes can be understood in terms of a sharp increase of the exciton-phonon entanglement.

We also note that this form of heteroentanglement is complementary to the superradiant behavior of the excitonic system. Superradiance (coherent spontaneous emission) is the enhanced radiative decay compared to that of a monomer as a result of the coherent nature of the electronic excited states. $^{21,22}$ If all transition dipoles of the $N$ monomers are parallel, superradiance of the $K=0$ state can be calculated from

$$
S:=\sum_{m n}\left(\rho_{e}^{K=0}\right)_{m n}
$$

It follows that excitonic superradiance and the excitonphonon heteroentanglement are complementary. For example, on the vertical $J=0$ axis in Fig. 2, the linear heteroentanglement (8) is at its maximum, $1-N^{-1}$, while the corresponding $K=0$ superradiance reaches its minimum value 1 ; on the horizontal $g=0$ axis, the linear heteroentanglement vanishes while corresponding $K=0$ superradiance reaches its maximum value $N$. For a given transfer integral $J$, as the exciton-phonon coupling $g$ is reduced, the superradiance gains while the exciton-phonon heteroentanglement decreases. Superradiance can be regarded as a form of the quantum coherence between different components of the exciton wave function localized on different sites. This coherence, in turn, can be viewed as a form of entanglement between spatially distinct excitonic modes (for a definition of mode entanglement, see, e.g., Ref. 23). Thus, the aforementioned complementarity phenomenon can be viewed as a form of entanglement transfer from the interexciton-phonon type to the intraexciton mode entanglement, and vice versa.

The role of off-diagonal exciton-phonon coupling as well as analyses of entanglement of polaronic states with nonzero crystal momenta are the subject of ongoing investigations. The Toyozawa Ansatz is again well-suited to capture the underlying physics in the presence of off-diagonal excitonphonon coupling. We believe that the results presented in this paper exemplify the fact that implementations of notions and apparatuses drawn from the emerging field of quantum information science are invaluable for gaining fresh insights for classic problems in condensed matter physics.

\section{ACKNOWLEDGMENTS}

Support from the Hong Kong Research Grant Council (RGC Competitive Earmarked Research Grant Award HKU 7010/03P) and the Committee for Research and Conference Grants of the University of Hong Kong is gratefully acknowledged. P.Z. thanks Z. D. Wang for the invitation and hospitality at the Physics Department of University of Hong Kong.
${ }^{1}$ L.D. Landau, Phys. Z. Sowjetunion 3, 644 (1933).

${ }^{2}$ S.I. Pekar, J. Phys. (Moscow) 10, 341 (1946).

${ }^{3}$ M. Horodecki, P. Horodecki, and R. Horodecki, in "Quantum Information-Basic Concepts and Experiments," edited by G.
Alber and M. Weiner (Springer, Berlin, 2000).

${ }^{4}$ For reviews, see D.P. Di Vincenzo and C. Bennet, Nature (London) 404, 247 (2000); A. Steane, Rep. Prog. Phys. 61, 117 (1998). 
${ }^{5}$ T.J. Osborne and M.A. Nielsen, Phys. Rev. A 66, 032110 (2002); A. Osterloh, L. Amico, G. Falci, and R. Fazio, Nature (London) 416, 608 (2002); G. Vidal, J.I. Latorre, E. Rico, and A. Kitaev, Phys. Rev. Lett. 90, 227902 (2003); T.-C. Wei, D. Das, S. Mukhopadyay, S. Vishveshwara, and P.M. Goldbart, quant-ph/ 0405162 (unpublished).

${ }^{6}$ G. Vidal, Phys. Rev. Lett. 91, 147902 (2003); G. Vidal, quantph/0310089 (unpublished); M. Zwolak and G. Vidal, cond-mat/ 0406440 (unpublished); F. Verstraete, J.J. Garcia-Ripoll, and J.I. Cirac, cond-mat/0406426 (unpublished).

${ }^{7}$ R.W. Munn, and R. Silbey, J. Chem. Phys. 83, 1843 (1985); 83, 1854 (1985).

${ }^{8}$ Y. Zhao, D.W. Brown, and K. Lindenberg, J. Chem. Phys. 100, 2335 (1994); 106, 2728 (1997).

${ }^{9}$ S. Ishihara and N. Nagaosa, Phys. Rev. B 69, 144520 (2004).

${ }^{10}$ T. Holstein, Ann. Phys. (N.Y.) 8, 325 (1959); 8, 343 (1959).

${ }^{11}$ A. Matsui and K. Mizuno, 5th Intern. Conf. on Excited States in Solids, Lyon, 1985.

${ }^{12}$ D. Emin, Adv. Phys. 22, 57 (1973).

${ }^{13}$ Y. Toyozawa, Prog. Theor. Phys. 26, 29 (1961).

${ }^{14}$ Y. Zhao, Doctoral thesis, University of California, San Diego, 1994.
${ }^{15}$ Y. Zhao, D.W. Brown, and K. Lindenberg, J. Chem. Phys. 107, 3159 (1997); 107, 3179 (1997); 106, 5622 (1997); A. Romero, D.W. Brown, and K. Lindenberg, ibid. 109, 6540 (1998).

${ }^{16}$ V.M. Buinmistrov and S.I. Pekar, Sov. Phys. JETP 5, 970 (1957).

${ }^{17}$ E. Jeckelman and S.R. White, Phys. Rev. B 57, 6376 (1998).

${ }^{18}$ J. Bonca, S.A. Trugman, and I. Batistić, Phys. Rev. B 60, 1633 (1999).

${ }^{19}$ A. Renyi, Proc. Fourth Berkeley Symp. Math. Stat. Prob. 1960 (University of California Press, Berkeley, 1961), Vol. I, p. 547.

${ }^{20}$ Y. Zhao, P. Zanardi, and G.H. Chen (unpublished).

${ }^{21}$ Y. Zhao, T. Meier, W.M. Zhang, V. Chernyak, and S. Mukamel, J. Phys. Chem. B 103, 3954 (1999); T. Meier, Y. Zhao, V. Chernyak, and S. Mukamel, J. Chem. Phys. 107, 3876 (1997); Y. Zhao, G.H. Chen, and L. Yu, ibid. 113, 6502 (2000).

${ }^{22}$ M. Lippitz, C.G. Hubner, T. Christ, H. Eichner, P. Bordat, A. Herrmann, K. Mullen, and T. Basche, Phys. Rev. Lett. 92, 103001 (2004); S.H. Lim, T.G. Bjorklund, F.C. Spano, and C.J. Bardeen, ibid. 92, 107402 (2004); F. Meinardi, M. Cerminara, A. Sassella, R. Bonifacio, and R. Tubino, ibid. 91, 247401 (2003).

${ }^{23}$ P. Zanardi, Phys. Rev. A 65, 042101 (2002). 